\title{
EL "POLICUBO" COMO HERRAMIENTA DIDÁCTICA PARA FAVORECER EL PROCESO DE ENSEÑANZA-APRENDIZAJE DE LA CONSTRUCCIÓN INDUSTRIALIZADA
}

\section{THE "POLICUBE" AS A TEACHING TOOL TO FAVOR THE TEACHING LEARNING PROCESS OF INDUSTRIALIZED CONSTRUTION}

\author{
Douthat, Maira Lucía ${ }^{1}$, Longa, Lorena Beatriz², Gauna, Mauricio Martín³, Morán, Rosanna Griselda ${ }^{4}$ \\ 1 ITDAHu - FAU - UNNE, Resistencia, Chaco, Argentina, 2 ITDAHu - FAU - UNNE, Resistencia, \\ Chaco, Argentina, 3 ITDAHu - FAU - UNNE, Resistencia, Chaco, ArgentinA, 4 ITDAHu - FAU - \\ UNNE, Resistencia, Chaco, Argentina. \\ mairadouthat@gmail.com
}

\section{RESUMEN}

La asignatura Construcciones II "A" de la Facultad de Arquitectura y Urbanismo de la Universidad Nacional del Nordeste aborda la problemática del diseño de sistemas constructivos no convencionales: prefabricación no integral, prefabricación integral y la aplicación de sistemas alternativos ("Ballon Frame" y "Steel Frame") y contenedores metálicos.

El objetivo de este trabajo consiste en analizar el conjunto de viviendas llamado "Kubuswoningen", localizado en Rotterdam, Holanda y sus posibles aplicaciones en el desarrollo proyectos creativos para utilizar la idea como disparador de la motivación de los estudiantes, estimulándolos en el proceso de diseño, y analizar también los principios geométricos constructivos del "policubo", que permiten reconocer nuevas formas de responder a la demanda habitacional a la vez que se priorizan los espacios urbanos, recurriendo a la industrialización como herramienta constructiva.

La metodología de análisis es de carácter descriptivo, teniendo como base el estudio de obras diseñadas a partir del policubo.

Basados en dicho análisis, vemos que la utilización de una modulación puede generar trabajos innovadores en el desarrollo de proyectos de construcción industrializada, explorar nuevas formas de construir y de considerar al hábitat humano, de forma tal que responda a las necesidades humanas y ambientales para fomentar su difusión y desarrollo local.

\section{ABSTRACT}

The subject Constructions II "A" of the Faculty of Architecture and Urbanism of the National University of the Northeast addresses the problem of the design of unconventional construction systems: nonintegral prefabrication, integral prefabrication and the application of alternative systems ("Ballon Frame" and "Steel Frame") and metal containers.

The objective of this work is to analyze the housing complex called "Kubuswoningen", located in Rotterdam, Holland and its possible applications in the development of creative projects to use the idea as a trigger for student motivation, stimulating them in the design process, and also analyze the geometric constructive principles of the "polycube", which allow us to recognize new ways of responding to housing demand while prioritizing urban spaces, using industrialization as a constructive tool.

The analysis methodology is descriptive, based on the study of works designed from the polycube.

Based on this analysis, we see that the use of a modulation can generate innovative works in the development of industrialized construction projects, explore new ways of building and considering the human habitat, in a way that responds to human and environmental needs to promote its diffusion and local

development. 
Douthat, Maira Lucía ${ }^{1}$, Longa, Lorena Beatriz ${ }^{2}$, Gauna, Mauricio Martín ${ }^{3}$, Morán, Rosanna Griselda ${ }^{4}$

PALABRAS CLAVES: Industrialización, Diseño, Prefabricación, Modulación, Innovación.

KEY WORDS: Industrialization, Design, Prefabrication, Modulation, Innovation.

\section{INTRODUCCIÓN}

La problemática de la asignatura Construcciones II "A" se centra en la aplicación del método industrial de producción en el campo específico de la arquitectura, a cuyo efecto se estudian los fundamentos de la Coordinación Modular como base para la fabricación de los componentes de la construcción, sustentada en los principios de la industria: racionalización del trabajo, normalización constructiva y tipificación productiva.

Planteado así el problema, se aplican criterios de Arquitectura Modular (AM), que consiste en el diseño y manejo de sistemas compuestos por elementos constructivos industrializados, diseñados con dimensiones modulares coordinadas, con capacidad de conectarse entre sí, y ser ubicados en distintas posiciones y situaciones en obra, ser reemplazados o intercambiados, sin que ello afecte al sistema, ni provoque una significativa merma en los estándares de calidad del producto final diseñado. Se logra así una arquitectura versátil y personalizada, con grandes ventajas en el momento de su materialización y en la protección del ambiente donde se implanta, con gran variedad de uso, acotados tiempos de producción y mínima huella ecológica, a través de la reducción de desperdicios de obra.

La "Teoría de los Policubos" tiene íntima vinculación con las matemáticas recreativas que, mediante la manipulación de los policubos, brindan las bases para el desarrollo de habilidades creativas, visuales y verbales, por las experiencias espaciales que se pueden encontrar en ellos. Dada la condición volumétrica de los policubos, a partir de su estudio se pueden generar formas simples y complejas, razón por la que son preferentemente utilizados en el proceso de aprendizaje debido al aporte que proporcionan al desarrollo del pensamiento geométrico, que es la base para el entendimiento del entorno en el cual vivimos.

El presente trabajo plantea el análisis de dos conceptos compatibles, la Arquitectura Modular y la Teoría de los Policubos, tomando sus criterios básicos, la clasificación y las posibilidades de agrupación, estableciendo correspondencias con representaciones de uso arquitectónico, como una manera de incentivar a los estudiantes en el proceso creativo de diseño, explorando otras formas de abordaje, dadas las múltiples ventajas que poseen aportando resultados para la creación de estructuras capaces de satisfacer los diversos requerimientos del proyecto.

Como unidad de análisis se tomó la obra del arquitecto Piet Blom, conocida como "Kubuswoningen" ("Casas Cubo"), ubicada en la ciudad de Rotterdam, en Holanda.

Esta obra nos ayuda a comprender de manera clara y precisa la relación intrínseca entre ambas teorías, verificando cómo impacta cada una en la forma de diseñar, según sea el sitio donde se emplaza y la sociedad que lo usa, lo que puede considerarse un hito en la arquitectura mundial.

\section{METODOLOGÍA}

La metodología de análisis es de carácter descriptivo, teniendo como base el estudio de obras diseñadas a partir del policubo. 
Douthat, Maira Lucía ${ }^{1}$, Longa, Lorena Beatriz ${ }^{2}$, Gauna, Mauricio Martín ${ }^{3}$, Morán, Rosanna Griselda ${ }^{4}$

\section{DESARROLLO}

\section{Teoría de los Policubos}

La teoría de los policubos nace a partir de la teoría de los poliminós, que son "un conjunto de cuadrados iguales unidos por sus lados, de tal manera que cada dos de ellos mantienen al menos un lado en común" (Quezada Feijoó, 2012, p.17).

La idea fue propuesta por el ingeniero y matemático Salomón W. Golomb (1954), y más adelante se la reconoció como una rama de la geometría combinatoria. Los cuadrados conectados entre sí se clasifican de acuerdo a su número: monominós (un solo cuadrado), dominós (dos cuadrados), triminós (tres cuadrados), tetraminós (cuatro cuadrados), pentaminós (cinco cuadrados), n-minós (formados por n-cuadrados).

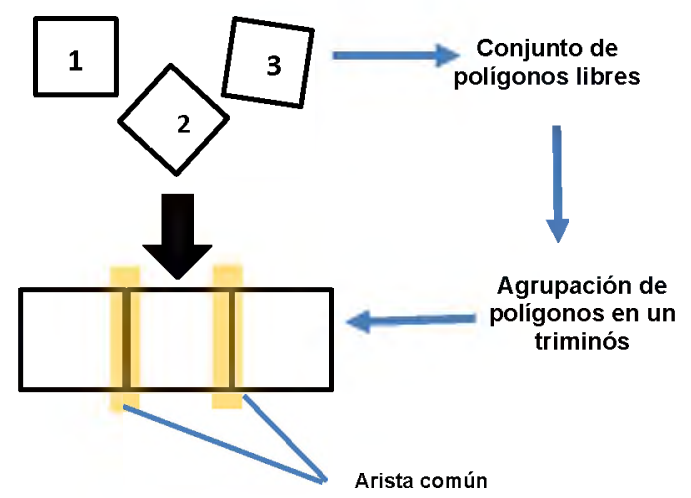

Fig. 1: Conformación de poliminós. Fuente: elaboración propia en base a Quezada Feijoo, 2012.

También se los clasifica, según la forma que se obtiene de su agrupación, en regulares, irregulares, cóncavos, convexos, y también como formas libres o contenidas, según la libertad de agrupación que tengan los cuadrados entre sí. Se determina así una cuantiosa variedad de posibilidades que pueden lograrse de su agrupación. Hasta aquí las combinaciones logradas en el plano bidimensional. Del mismo modo, en el espacio también se logra una significativa variedad de combinaciones denominadas "policubos".

\section{Policubos}

"Un policubo es una generalización tridimensional del concepto de poliminó, que consiste en un conjunto de módulos cuadrados unitarios unidos por sus lados. La teoría de policubos es una rama de las matemáticas que se ocupa de estudiar el comportamiento de unidades modulares cúbicas, tal que unidas por sus caras configuran formas en el espacio tridimensional". (Quezada Feijoó, 2012, p.21).

Al igual que los poliminós, las posibilidades de agrupación en los policubos es infinita, cuanto mayor sea el número de cubos, mayores serán las variantes que podamos obtener, resultando en una gran variedad de módulos que conservan ortogonalidad entre sus caras $y$, dentro de la sencillez de sus formas, aportan riqueza volumétrica y modularidad, correspondiéndose a formas aptas para su uso arquitectónico.

Una vez elegido el cubo de estas dimensiones, se analizaron las más importantes y conocidas disecciones que existen y que son consideradas rompecabezas geométricos. Estos son: el Cubo de 
Douthat, Maira Lucía ${ }^{1}$, Longa, Lorena Beatriz² , Gauna, Mauricio Martín ${ }^{3}$, Morán, Rosanna Griselda

Conway, el Cubo O' Berine, el Cubo Diabólico, el Cubo 7, el Cubo Soma, el Cubo de Nob, el Cubo de Coffin, el Cubo de Lola, el Cubo de Steinhaus y el Cubo Mikusisnki (Fig.2).

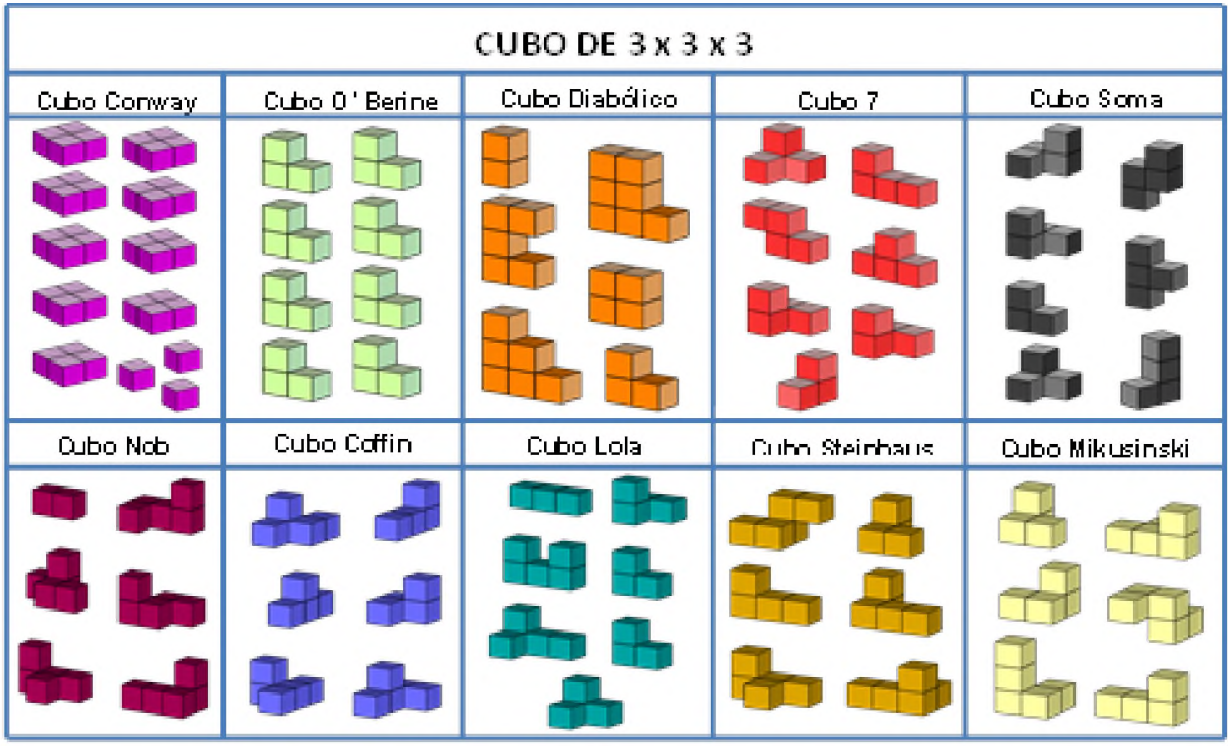

Fig. 2: Disecciones de cubos de 3×3×3. Fuente: (Quezada Feijoó, 2012, Anexo 2).

De acuerdo a las formas que se obtuvieron de la combinación, por la libertad de agrupación que se les proporcionó, se analizó la construcción de cubos con policubos (cubos contenidos), tomando como referencia el caso particular del cubo de $3 \times 3 \times 3$, por el número adecuado de piezas policúbicas que contiene cada una de sus disecciones.

Una variante muy conocida de este tipo de organizaciones espaciales a partir de cubos es la propuesta del "Módulo HELE" del Arq. Rafael Leoz de la Fuente (1965). El Módulo HELE fue el inicio de una propuesta de división y ordenamiento del espacio arquitectónico por medio de principios geométricos. Ver Fig. 3.

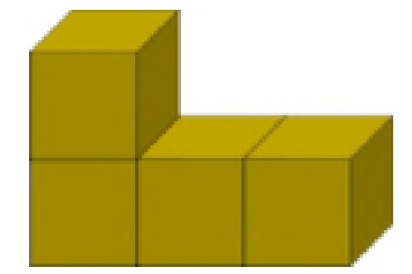

Fig. 3: Modulo HELE. https://www.google.com.ar/search?q=modulo\%20hele\&tbm=isch\#imgrc=smShJNPytHt1yM

\section{Clasificación de policubos}

Además de la clasificación ya mencionada, se los puede clasificar como:

Policubos libres: El conjunto de cubos conectados poseen la libertad de expansión a diferentes lugares, carecen de contenedor que pueda limitar su agrupación, y pueden formar estructuras orgánicas.

Policubos contenidos: El conjunto de cubos rellenan por completo un prisma, considerado como el contenedor que limita la agrupación hasta alcanzar su forma definitiva sin dejar espacios intermedios. Hay ejemplos donde se la combinación de ambas configuraciones: libres y contenidas, cuando al prisma contenedor se lo rellena dejando aberturas en su composición, logra la forma del prisma sin que ésta constituya una agrupación maciza en su totalidad. De este modo, los policubos poseen libertad de agrupación dentro de un contenedor limitante. 
Douthat, Maira Lucía ${ }^{1}$, Longa, Lorena Beatriz² ${ }^{2}$ Gauna, Mauricio Martín ${ }^{3}$, Morán, Rosanna Griselda

\section{Modulación}

Al trabajar con la Teoría de los Policubos, cuyo elemento es el cubo, estamos definiendo un módulo de composición, ya que estas formas poseen un carácter modular interesante al configurar espacios tridimensionales macizos que se acoplan perfectamente por sus lados y, aun cuando se sustituya uno de ellos, el conjunto conserva la misma organización.

Para utilizar este tipo de organización espacial en arquitectura, necesitamos definir las medidas con las que se trabajará, para lo cual se aplican los principios de la "Coordinación Modular", que simplifica y coordina las dimensiones de los elementos de construcción a ser ensamblados, mediante medidas comunes y sus múltiplos, a fin de lograr un máximo de eficiencia en los procesos de diseño y construcción de edificios, mediante la cual la arquitectura adquiere rasgos distintivos de diseño industrial, conciliando exigencias funcionales, técnicas y estéticas del producto, con medios y procesos de producción.

La Coordinación Modular exige que todas las dimensiones de los componentes del edificio estén relacionadas entre sí, es decir coordinadas, cuya unidad de medida y factor numérico es conocida con el nombre de MÓDULO.

El Módulo Básico (Fig.4), se denomina con la letra M y su valor internacional (normas IRAM) está normalizado en $10 \mathrm{~cm}$, por lo tanto $10 \mathrm{~cm}$ equivalen a $1 \mathrm{M}$.

MÓDULO BÁSICO

$(1 \mathrm{M}=1 \mathrm{dm}=10 \mathrm{~cm})$

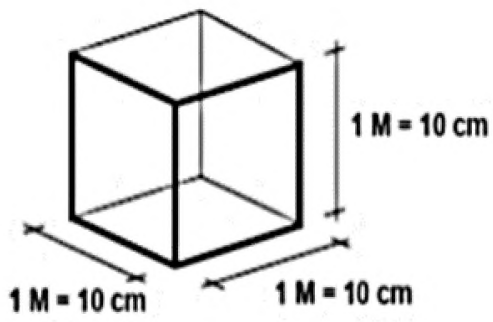

Fig. 4: Módulo básico: 10cm. Fuente: Publicación didáctica cátedra Construcciones II A

El módulo es el nexo dimensional entre el diseño y la construcción, que permite asegurar: la disminución de la variedad de las dimensiones de los componentes constructivos y la correlación de las medidas entre ellos; su intercambiabilidad y aditividad con el máximo número de combinaciones posibles; y la eliminación de desperdicios, cortes y ajustes ejecutados en obra. El módulo es aplicable en todas las etapas del proyecto y constituye la base dimensional de donde derivan los tamaños de las distintas cuadrículas modulares: cuadriculado de planos, estructuras, de obra, de urbanismo, etc. La utilización de la teoría de los policubos en el proceso de diseño, permite reconocer que existen principios ordenadores que organizan la composición proyectada, verificando que existen formas y espacios que satisfacen el desarrollo de las actividades que alberga el edificio proyectado.

Desde el punto de vista formal, es válida cualquier composición que resulte de adoptar un patrón y someterlo a alguna operatoria de simetría en el plano o en el espacio (incluyendo traslación, rotación, espejo, cambio de escala y todas sus combinaciones). En el caso de los policubos es una organización de cubos conectados por sus caras. Las piezas de un Cubo Soma, por ejemplo, constituyen patrones de repetición que pueden ser usados libremente para componer formas y asignarles una función.

Desde el punto de vista funcional, existen principios de organización de las partes que albergan actividades, respondiendo a condicionantes y requerimientos arquitectónicos previamente definidos. Si partimos de un vocabulario básico de organización (central, lineal, radial, etc.) y de los principios ordenadores (ejes, simetría, jerarquía, ritmo, etc.) es posible asociar la oferta de formas con la función 
Douthat, Maira Lucía ${ }^{1}$, Longa, Lorena Beatriz² , Gauna, Mauricio Martín ${ }^{3}$, Morán, Rosanna Griselda

asignada a cada espacio, por lo cual es fundamental analizarlas previamente para adecuarlas al tipo de organización y sus características.

La "Casas Cubo", presenta características concretas tratadas en la cátedra Construcciones II " $A$ " que se centra en la aplicación del método industrial de producción en el campo específico de la arquitectura, a cuyo efecto se estudian los fundamentos de la Coordinación Modular como base para la fabricación de los componentes de la construcción, sustentada en los principios de la industria: racionalización del trabajo, normalización constructiva y tipificación productiva.

\section{Caso real: Kubuswoningen o Casas Cubo (Rotterdam, Holanda, 1978-1984)}

Las Kubuswoningen, son un grupo de viviendas diseñadas por el arquitecto holandés Piet Blom. En 1977 recibió el encargo de estudiar el área del Oude Haven (Puerto Viejo) de Rotterdam con el objetivo de revitalizar la zona, que permanecía sin explotar desde la Segunda Guerra Mundial, y generar espacios residenciales dando prioridad a la vivienda social.

La idea del equipo técnico municipal era la de construir un puente peatonal sobre una de las principales arterias (Fig.5), pero que incluyera viviendas y servicios. El complejo contó con 38 Casas Cubo, espacios comerciales, una escuela, un edificio de apartamentos y una torre residencial, conocida como Het Potlood (El Lápiz).

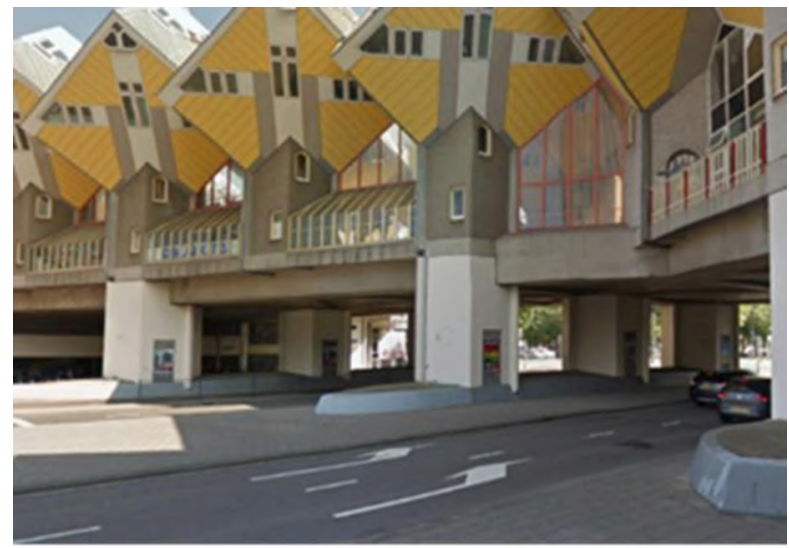

Fig.5: Localización y vista del proyectos. Fuente: https://www.archdaily.com/482339/ad-classics-kubuswoningen-piet-blom

Las Casas Cubo fueron diseñadas asimétricamente para semejar un bosque abstracto, cada techo triangular representando la copa de un árbol. Los edificios se construyeron sobre pilares de hormigón (tronco del árbol), con un entramado de madera (copa del árbol).

Fuste o tronco del árbol: la base del fuste tiene la forma de un hexágono y está construido a partir de tres columnas de concreto de carga con paredes de ladrillo de hormigón de gas en el medio. Las escaleras a lo largo del lado conducen a la entrada (2do piso).

Cubo o copa del árbol: el cubo está inclinado, aparentemente en equilibrio sobre su fuste, de modo que tres lados miran hacia el suelo y tres hacia el cielo. El ángulo entre los pisos y las paredes es de 53.5 grados (o el ángulo complementario), por lo que no es de 45 grados, por lo que este es un cubo algo aplastado (Fig. 6). Los pisos y las columnas portantes están hechos de concreto reforzado. 
Douthat, Maira Lucía ${ }^{1}$, Longa, Lorena Beatriz ${ }^{2}$, Gauna, Mauricio Martín ${ }^{3}$, Morán, Rosanna Griselda ${ }^{4}$

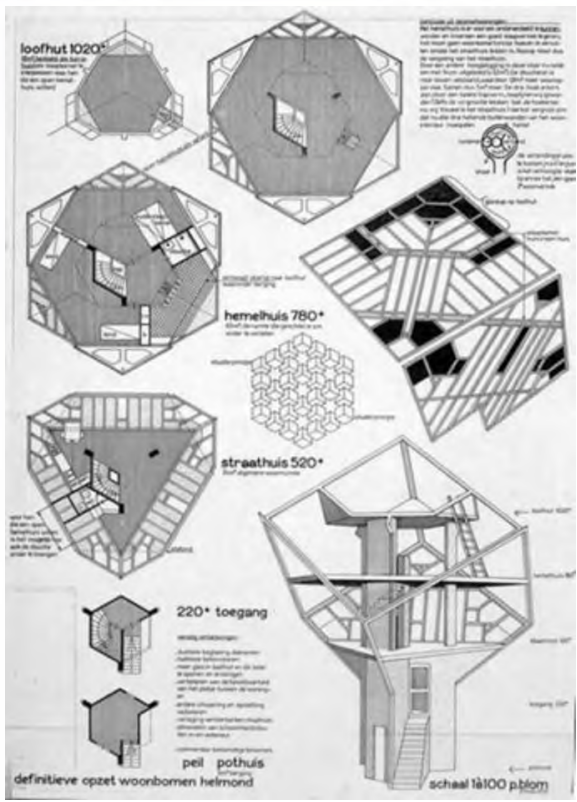

Fig. 6: Planos de Casa Cubo. Fuente: https://ar.pinterest.com/pin/78813062205542155/

El cubo consiste en un esqueleto de madera, que se une a los bordes del piso de concreto (Fig.7). Los paneles de cemento se atornillan en este esqueleto tanto en el interior como en el exterior. En el medio, hay lana de roca para el aislamiento. Todas las ventanas del cubo contienen doble acristalamiento. La primera capa de vidrio de las ventanas que miran hacia abajo es de $12 \mathrm{~mm}$ de espesor.

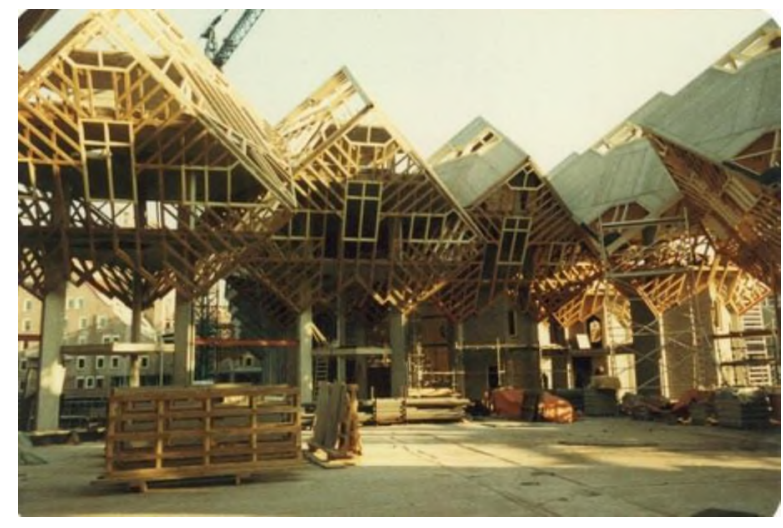

Fig. 7: Esqueleto de madera Casa Cubo. Fuente: http://www.kubuswoning.nl/woningen.html

El cubo tiene tres niveles diferentes, con un total de 100 metros cuadrados aproximadamente, aunque una cuarta parte del espacio es inhabitable. Las casas cubo tienen una altura de 22 metros (sin contar el pilar hexagonal) y cada uno de sus lados mide 7,5 metros.

La planta baja es la zona de entrada, el primer nivel posee un área triangular se utiliza como sala de estar y cocina abierta. Piet Blom llamó a este nivel "callejón» debido a que las ventanas dirigidas hacia abajo permiten una conexión visual con lo que está sucediendo en la calle. (Fig.8) 
Douthat, Maira Lucía ${ }^{1}$, Longa, Lorena Beatriz² ${ }^{2}$ Gauna, Mauricio Martín ${ }^{3}$, Morán, Rosanna Griselda ${ }^{4}$

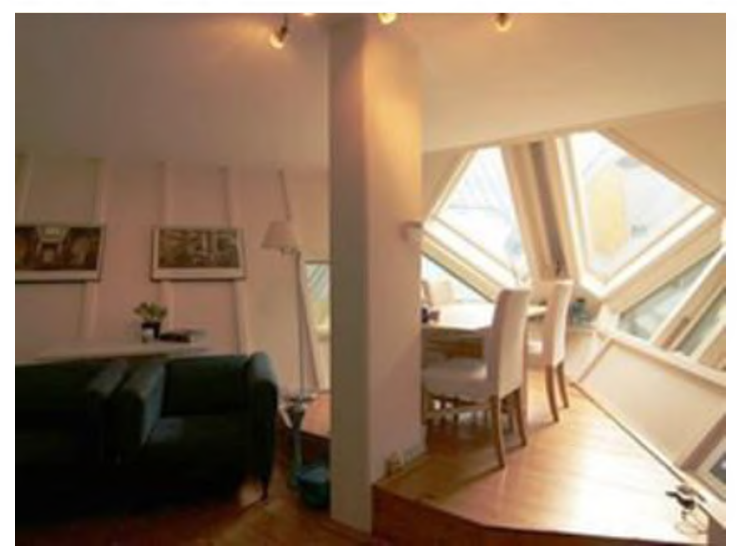

Fig. 8: Imagen interior primer nivel

Fuente: http://www.zoover.es/paises-bajos/holanda-meridional/rotterdam/kubuswoningen/fotos

El segundo piso con sus ventanas orientadas hacia arriba fue bautizado como la " casa celestial ", por el arquitecto, es la zona de dormir, con dos dormitorios, un pequeño salón y el baño. (Fig. 9)

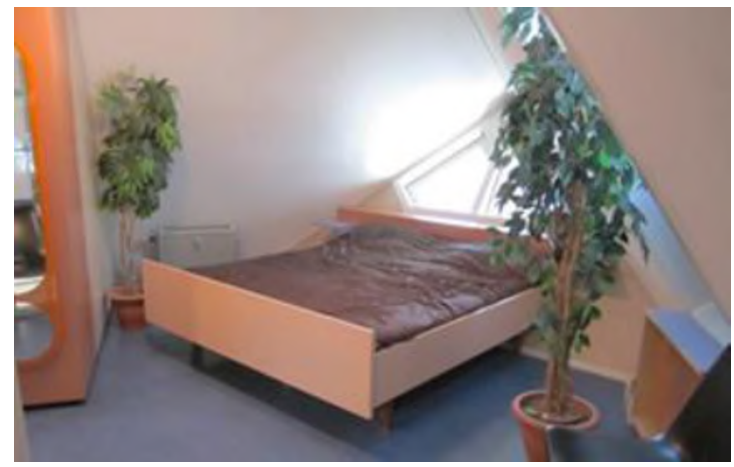

Fig. 9: Imagen interior segundo nivel.

Fuente: http://www.zoover.es/paises-bajos/holanda-meridional/rotterdam/kubuswoningen/fotos

Una plataforma con almacenamiento debajo y una escalera dan acceso al piso superior, el tabernáculo según Blom, una pirámide de tres lados con 18 ventanas y 3 escotillas que dan una amplia vista de los alrededores. Este espacio se puede utilizar como dormitorio, sala de niños o solario, dependiendo de las necesidades del habitante.

\section{DISCUSIÓN DE RESUTADOS}

Como la Teoría de los Policubos asociada a la Arquitectura Modular será incorporada a partir del ciclo lectivo 2020 como un aporte innovador en la asignatura Construcciones II A para la elaboración de los trabajos prácticos, confiamos en que el análisis realizado y de acuerdo a las conclusiones a las que hemos arribado, sea recibido de buen grado y aprovechado al máximo por los estudiantes, de forma clara y eficaz, pudiendo aplicar estos conceptos no sólo en los proyectos que realizarán en esta asignatura, sino a los que concebirán a lo largo de su carrera univestitaria y en el desarrollo de su vida profesional. 
Douthat, Maira Lucía ${ }^{1}$, Longa, Lorena Beatriz² ${ }^{2}$ Gauna, Mauricio Martín ${ }^{3}$, Morán, Rosanna Griselda

\section{CONCLUSIONES}

El desarrollo de la asignatura se articula en un Trabajo Práctico Integrador (TPI) que favorece especialmente el aprendizaje de los contenidos procedimentales. Su planteo, al inicio del cursado, sitúa al alumno en una condición de carencia, en la cual reconoce sus propios límites y hace que el desarrollo de los temas teóricos sea internalizados y acomodados de una forma más eficaz. En algunos puntos del desarrollo de esta, "la práctica antecede a la teoría", con el objetivo de favorecer el aprendizaje significativo sobre todos de los contenidos procedimentales. (Pilar y otros, 2016).

La aplicación de la Teoría de los Policubos y la Arquitectura Modular, pretende asignar a la materia un aporte específico en la etapa de diseño y modulación de los proyectos, con las siguientes ventajas:

- Permite ampliar el trayecto de diseño al recorrer un nuevo camino con la incorporación de desafíos basados en la creatividad para la materialización de los proyectos con un desarrollo tecnológicoconstructivos innovador.

- Abre un gran abanico de posibilidades de proyecto, debido a sus múltiples ventajas en cuanto a movimientos de intercambiabilidad y aditividad se refiere, logrando un máximo de combinaciones posibles y la disminución de variables dimensionales, evitando desperdicio de materiales y de tiempos de ejecución, lo que se traduce en una mayor rentabilidad.

- Incorpora una herramienta pedagógica innovadora centrada en la Teoría de los Policubos y la Arquitectura Modular, fortaleciendo el proceso de enseñanza de la construcción industrializada, y obteniendo riqueza volumétrica, modular, espacial y de confort en las configuraciones obtenidas sin perder la creatividad.

El presente análisis constituye un claro ejemplo de la factibilidad de incorporar propuestas creativas, utilizando aquellos materiales que ofrece el mercado aplicando innovaciones tecnológicas, posibilitando el traspaso de estos conocimientos a las nuevas generaciones de futuros profesionales y propiciando la evolución constante en el arte de hacer Arquitectura.

\section{BIBLIOGRAFÍA}

Caporioni, Garlatti, Tenca-Montini (1971): La Coordinación Modular - Madrid (España): Edit. Gustavo Gili.

Pilar, C.; Morán, R.; Vedoya, D. (2016). Sistemas constructivos industrializados para resolución de equipamientos en situaciones de catástrofe. Revista ARQUITECNO Nº 8. Noviembre 2016. Página 60 a 66. Corrientes (Argentina). Ediciones del ITDAHu.

Quezada Feijoó, E. D. (2012): Arquitectura modular basada en la teoría de policubos. Trabajo de fin de titulación, Universidad Técnica particular de Loja - Loja (Ecuador). Director: Arq. Xavier Burneo.

Serrentino R. y Molina, H. (2002): Arquitectura modular basada en la teoría de policubos - Caracas (Venezuela): SIGraDi nov.2002. pp. 264-267.

Vedoya, D. (2013): La transposición tecnológica como estrategia de diseño - Ctes (Arg.): Edic. del ITDAHu. Vedoya, D. (2014): La transposición tecnológica. Introducción a la génesis de los procesos tecnológicos Saarbrücken (Alemania): Editorial Académica Española.

Vedoya, D. E. (2016): La Coordinación Modular (Publicación Didáctica) - Cátedra de Construcciones II: Facultad de Arquitectura y Urbanismo (FAU), Universidad Nacional del Nordeste (UNNE).

\section{Páginas WEB consultadas}

www.jm3studio.com/casas-cubo-de-Rotterdam.

www.es. wikiarquitectura.com/edificio/casas-cubo.

http://www.zoover.es/paises-bajos/holanda-meridional/rotterdam/kubuswoningen/fotos

http://www.kubuswoning.nl/woningen.html

https://ar.pinterest.com/pin/78813062205542155/

https://nl.wikipedia.org/wiki/Kubuswoningen_(Rotterdam) 